\title{
Simulation and experimentation supplementing the teaching of basic concepts in optics
}

\section{Lia Zerbino, N. Baade, M. Lavagna}

Lia Maria Zerbino, N. N. Baade, M. E. Lavagna, "Simulation and experimentation supplementing the teaching of basic concepts in optics," Proc. SPIE 3831, Sixth International Conference on Education and Training in Optics and Photonics, (16 June 2000); doi: 10.1117/12.388750

Event: Education and Training in Optics and Photonics (ETOP'99), 1999, Cancun, Mexico 


\title{
SIMULATION AND EXPERIMENTATION SUPPLEMENTING THE TEACHING OF BASIC CONCEPTS IN OPTICS
}

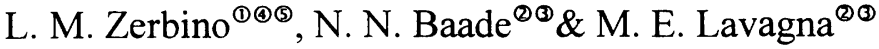 \\ IMApEC \& OPTIMO \\ Facultad de Ingeniería, Universidad Nacional de La Plata \\ Argentina \\ E-mail: liaz@odin.ciop.unlp.edu.ar
}

Keywords: Simulation, educational software, Fresnel Equations

\begin{abstract}
We present a teaching strategy combining computer simulation and laboratories as a way of introducing personalized teaching and considering new communication codes in order to engage students in a dynamic learning process, even in large groups at the university, focusing on a critical approach, adaptability to various kinds of languages and representations, continuous testing of the validity of their hypothesis, their limits and those of the models and solutions suggested, as a way of preparing individuals capable of generating and/or interpreting and applying the science of the third millennium. This is an ambitious idea and, in order to succeed in all that was mentioned above, non-conventional elements, like computers, were incorporated to the teaching process to get the students interested and to motivate them so that they can better interact. The introductory experience involves the design of software about Fresnel Equations and aims to solve conceptual problems that appear when assigning negative values to the amplitudes of electromagnetic waves (OEM) at an interface. Conceptual implications, like the analysis of changes of phase and amplitude, allow then the understanding of thin film interference phenomena, critical angle, polarization angle.... The program is interactive and designed in such a way that the user him/herself has to find out the conditions of the model that is being used, and to interpret and/or predict results on that basis. The experience is complemented with very simple laboratory equipment that allows for hands-on testing of the students' predictions.
\end{abstract}

\section{FOUNDATIONS}

We are working for a change in the educational paradigm, which implies a detailed revision of current teaching methodologies and curricula. If this change were not sought for, the University as an institution would not be assuming its main role: to be the leading actor and guide in a society demanding from citizens a continuous adaptation to scientific and technological evolution. The new professor-student profile should be built based on the respect for own idiosyncrasies as regards educational tasks.

In addition to this, "The great efficacy of meaningful learning as a means of processing and storing information may be attributed up to a great extent to two distinct characteristics: intentionatlity and sustaintionality of the learning task with the cognitive structure.

Firstly, when intentionally relating potential meaningful material with established and pertinent ideas from their cognitive structure, students are able to explore with full efficacy their knowledge as if it were an organising and ideative matrix in order to incorporate, understand, and fix new ideas.

It is the very intentionality of this process that enables students to use their previous knowledge as the basis to process new meanings (concepts, facts, principles,...)". (Ausubel).

In this context, it is natural to consider the advantages of introducing computers as didactic tools, and not only because of their generalised everyday use, which makes them acceptable and motivating. The importance of the implementation of an 
educational software simulating a model of physical phenomena to complement experiments in the laboratory is mainly due to its possibilities, such as:

a) animation or dynamic visualisation;

b) implementation of interactive methods;

c) easy and quick repetition of the process;

d) practice with the adaptability to different languages, symbols, and representations;

e) continuous verification of work hypothesis and validity limits of models and solutions;

f) speed of calculations and representation with different initial conditions and/or environment of the phenomena under study.

\section{OBJECTIVES}

In the case of teaching Optics, it is known that students find it difficult to conceptualise the different models (waves, rays, particles), and their correct utilisation for the interpretation and analysis of different luminous phenomena. Particularly in the case of wave optics, it would also be interesting to strengthen the abstractions involved in the model (amplitude, phase, frequency, polarisation), and to relate them to experimental observations (intensity, chromaticity, coherence). A deep comprehension of the wave model is sought for, while keeping it independent from the concept of light.

As a first step, we present a software dealing with Fresnel Equations to complement reflection and refraction experiments in different dielectric media, which allows:

a) to provide an animated visualisation of the wave model and of the propagation model of waves in different media, as well as its behaviour in the interfaces.

b) to solve erroneous conceptualisations on the part of the students, in the assignment of negative values to electromagnetic waves (OEM) amplitudes, a problem that arises in the interface of two dielectric media. (Students usually do not assume that "Fresnel equations are related to the specific directions of the fields from which they were deducted". Hecht-Zajac'.)

c) to relate the variables of the model (amplitude, phase) to observable phenomena during the experiment (intensities).

d) to compare the theoretical values of Fresnel coefficients with those obtained at the laboratory, thus testing the validity and limits of the model.

e) to predict and confirm cases, situations, and applications of technological interest.

\section{PRODUCTION AIMS}

The conceptual implications of this strategy, such as the analysis of phase change and the relationship between incident, reflected and transmitted wave amplitudes, will be the elements of the organising and ideative matrix proposed by Ausubel, since interference phenomena in dielectric films, critical angle, polarization angle, etc., can be understood from these concepts.

\section{CHARACTERISTICS OF THE SOFTWARE}

The software presents the problem in an interactive way, and it is prepared so as to make users interpret on their own the conditions of the model and predict behaviours and results of the propagation of electromagnetic waves (OEM) in different media.

\subsection{THEMATIC CONTENTS OF THE SOFTWARE}

a) Reflection and refraction phenomena with the luminous ray model, in light propagation.

b) Light propagation in different homogeneous, isotropic, and linear media, with the model of the wave.

c) Fresnel Equations for homogeneous, isotropic and linear media.

d) Phase changes and amplitude relations for waves.

e) Polarization by reflection.

f) Critical angle. 


\subsection{DESCRIPTION OF SOFTWARE SCREENS}

Main Screen: (Figure 1). It is interactive and consists of three fields:Controls, Data and Simulation

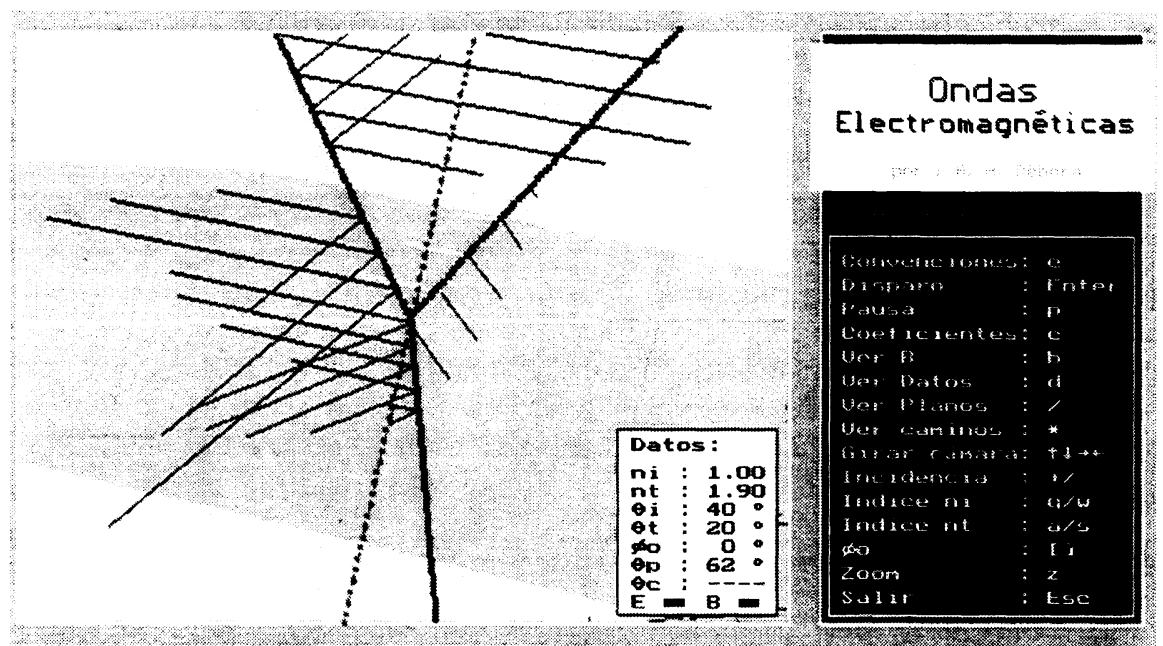

Figure 1

Controls (Figure 2)

a) of data: instructions to change data.

b) for visualisation:

* rotate camera: it changes the perspective of the image by rotating in the Simulation space.

zoom: it allows to enlarge the scale (Figure 1)

* stop: it freezes wave propagation at any given instant.

* shooting: it begins the simulation of the propagation in the simulation space.

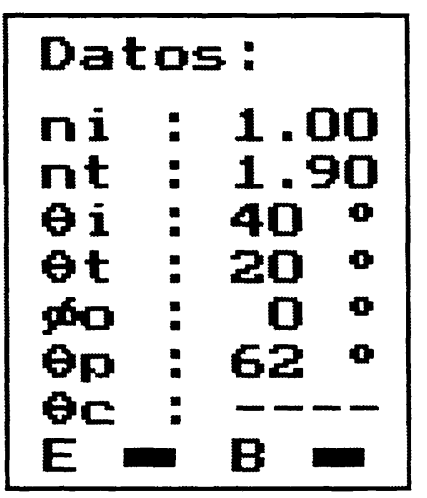

Figure 3

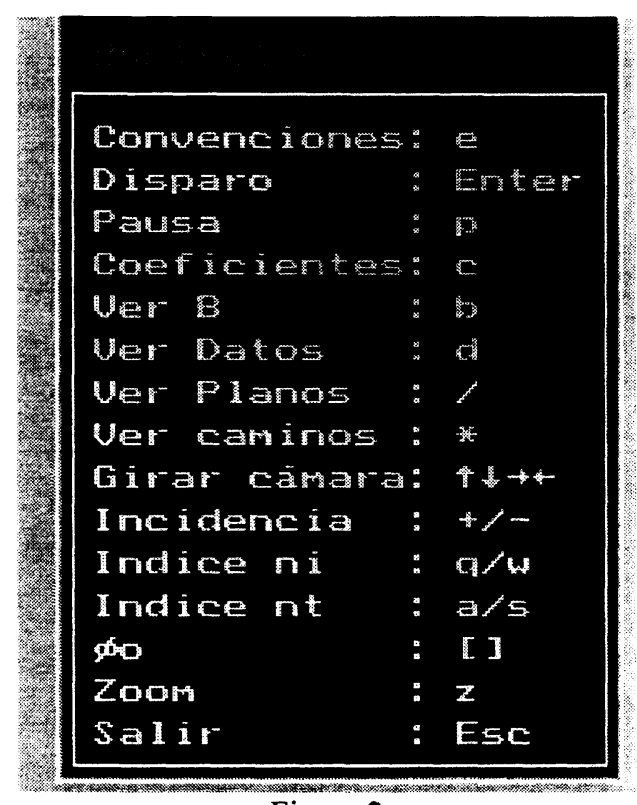

Figure 2

Data (Figure 3) Chart with:

* The values of the significant variables: ni, nt, $\theta \mathrm{i}, . \theta \mathrm{t}$, and $\phi 0$., which can be modified. They can be chosen with an accuracy of up to 2 decimals for the indexes and up to $5^{\circ}$ for the angles.

* The polarisation angle and the critical angle, determined by the programme according to the values set in 1 .

* The colours characterising the incident magnetic and electric fields vectors. 
Simulation: Space where the following are represented:

- Incidence plane

- Separation plane between the two media.

- ways: Propagation direction of the waves (optional visualisation). (Figure 4)

- planes: Planes perpendicular to the incidence plane and which contain the filed vectors (optional visualisation). (Figure 5)

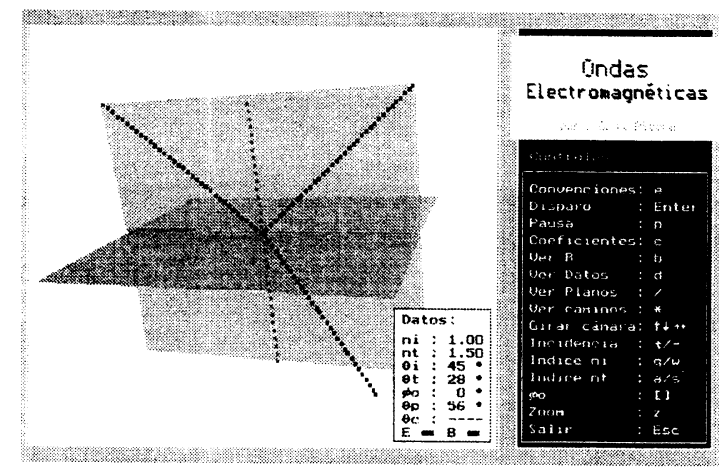

Figure 4

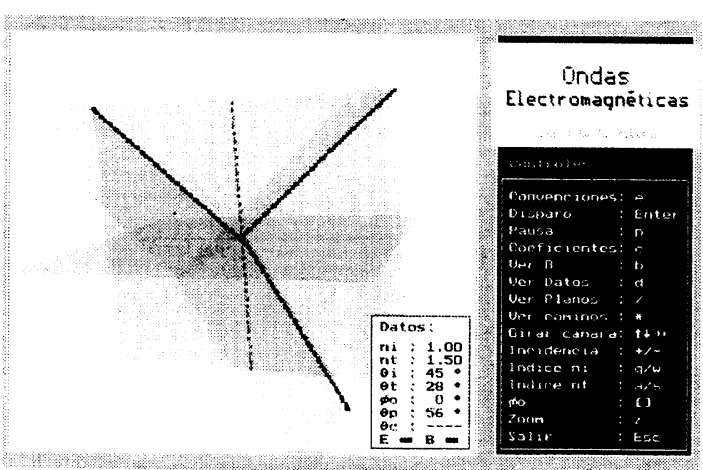

Figure 5

- Propagation of the incident electromagnetic wave and its corresponding reflected and transmitted waves when it reaches the interface: This is simulated by visualising the different positions that a set of vectors representing the different states of the electric field during a given period would take (optional visualisation beginning with the command shoot). (Sequence of Figures 6, 7, 1 and 8).

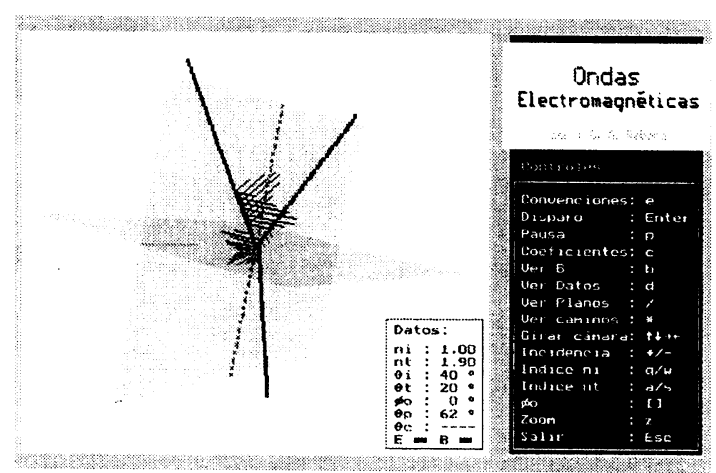

Figure 7

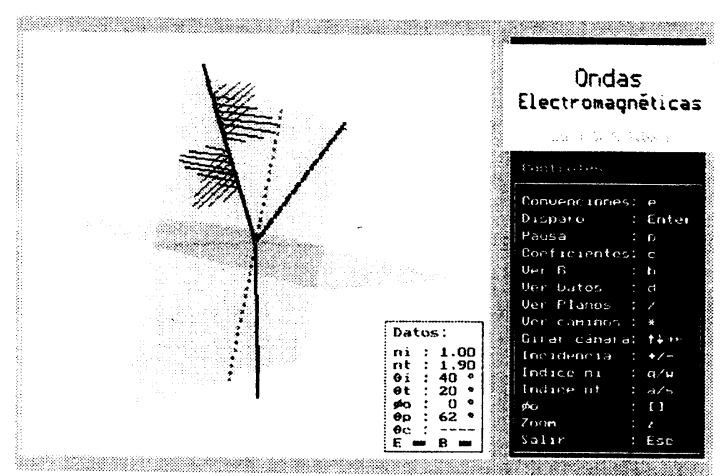

Figure 6

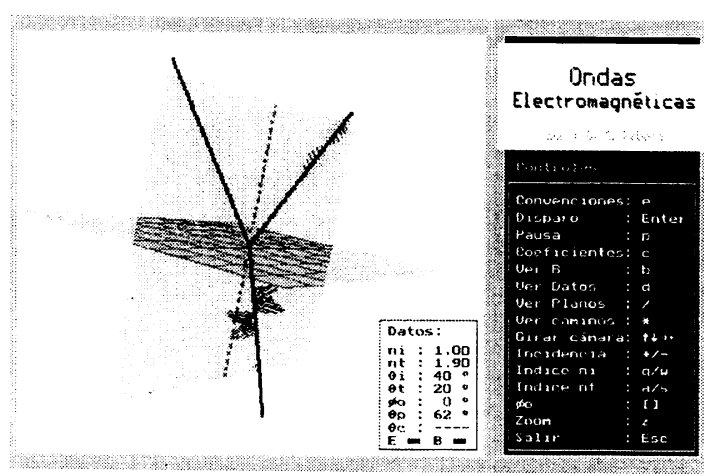

Figure 8 
Convention Screen: (Figure 9)

- Graphic showing the polarisation state of the components which are parallel and perpendicular to the incidence plane of the electric field vector, from which the Fresnel Equations used for the software programme were deduced. The polarisation of the magnetic field vector is not indicated, it is determined by means of Poynting vector.

- Definition of the angle $\phi_{0}$, determined by the direction of the electric field vector of the incident wave and the incidence plane.

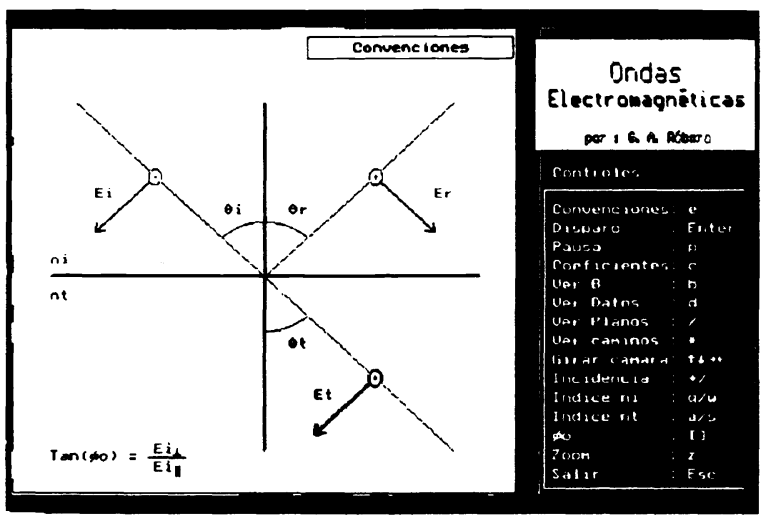

Fresnel Coefficients Screen: (Figure 10)

- Graphic representation of the amplitude coefficients according to the incidence angle. This representation for homogeneous, isotropic, and linear media both for internal and external reflection, is calculated and plotted by the software programme according to input data.

\section{METHODOLOGICAL PROPOSAL}

The set software-laboratory instrumental is integrated by means of study, development and evaluation Guides. Taking into

Figure 9

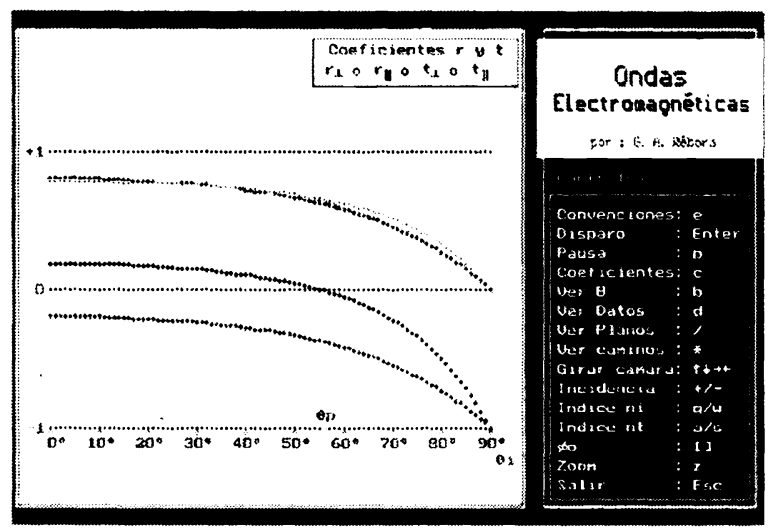

Figure 10 account that laboratory instrumental and time to spend at the laboratory are usually scarce, the usefulness of the simulation as a permanent support to answer different questions raised on the Guides and to verify the good judgements - or not - on the part of the students, is evident.

Even though the conception of software-experiments has intended to preserve the versatility needed to use them in the classroom, we propose a methodology successfully tested with small groups as an example.

a) simulation to visualise the behaviour of the electric field vectors of the incident, reflected and transmitted waves on an interface, for different incidence angles; to analyse the corresponding Coefficients screen qualitatively and identify the conditions of the change in sign; to fix those conditions on the main screen and relate phase changes to coefficients negative values.

b) mathematics software to calculate and represent the values of Fresnel Coefficients for reflection and of the reflectances for the interface between two media available for the experiment, for two different wavelengths.

c) experimentation to determine the values of the reflectances corresponding to different incidence angles and for different wavelengths. The experimental values obtained for reflectance are plotted, and the reflection coefficients for different wavelengths are calculated and plotted based upon those values.

d) theoretical and experimental graphics are compared, and the precision limits of the coefficients calculated through the simulation are discussed.

e) simulation to find the conditions to obtain special situations (polarisation through reflection). 
f) calculations are carried out in order to predict the conditions to obtain situations equivalent to e) for the materials with which the experiment was carried out.

g) the predicted conditions f) are experimentally reproduced and the results obtained are analysed.

It is evident that, with slight modifications, this strategy could begin with an experimental verification of particular situations for whose explanation and justification it would be interesting to use the software.

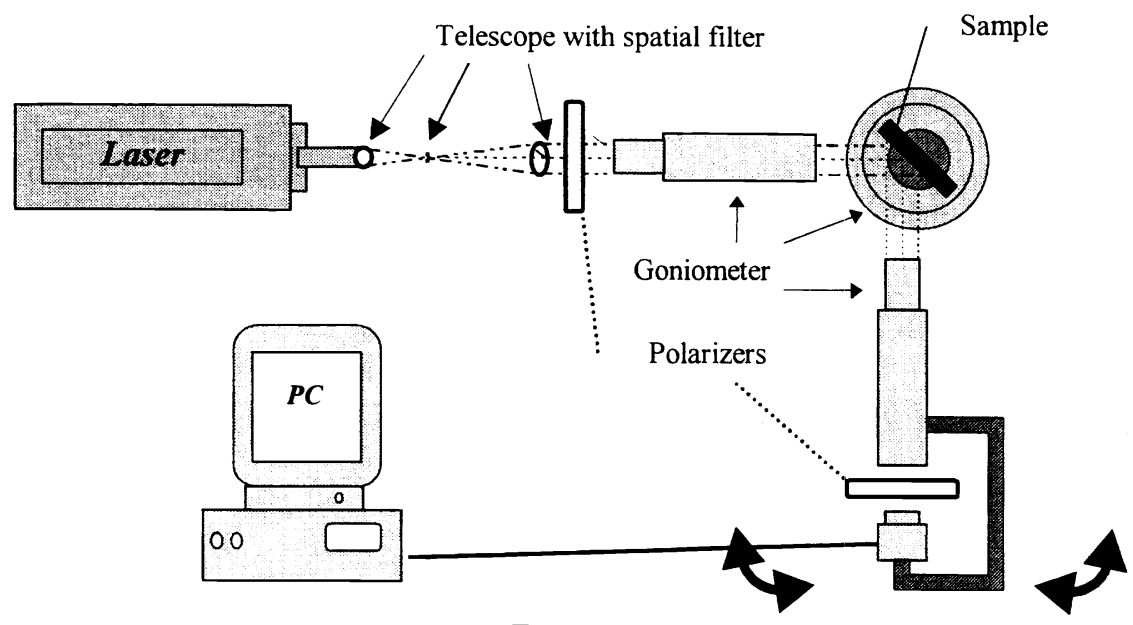

Figure 11

\section{EXPERIMENTAL DEVICE}

The minimum necessary instrumental for the implementation of this strategy is that commonly used at university courses of General Physics for the study of the laws of reflection and the verification of the existence of a critical angle. However, in order to apply this strategy to large groups, it was complemented with an automatic system of data acquisition that allows the optimisation of the use of classroom hours. Figure 11 shows a diagram of the device.

\section{CONCLUSIONS}

In university courses where the contents of basic physics are introduced, it should be a priority that research principles and techniques, although simple, be incorporated in a continuous way, so as to gradually contribute to the formation of the concept and to the development of skills.

Currently there have been changes in the way experiments are carried out: not only are we able to analyse in detail the results of experiments with a certain ease, but the possibilities to carry out the experiments themselves have increased, due to the possibility of analysing the information in databases and also to the possibility of a computerised control of the devices used.

We believe that this way of facing the learning-teaching process provides a starting point for the development of the methodological skills of information analysis required for the advancement in current sciences and technologies.

\section{REFERENCES}

1.- J. D. Novak y D. B. Gowin. Aprendiendo a aprender.

2.- E. Hecht / A. Zajac. Óptica.

\section{BIBLIOGRAPHY}

- Teodoro y J. C. Freitas. Desenvolvimientos de Sistemas Educativos. Educacao e Computadores. Lisboe: GEPME.

- J. Grayson and L.C. Mc Demmott. ( 1996 ). Use of the computer for research on student thinking in Physics. Am.J.Phys. 64(5), May 1996, 557-565.

- Erik De Corte. Aprendizaje apoyado en el computador: Una perspectiva a partir de Investigación acerca del aprendizaje y la Instrucción. Actas del Tercer Congreso Iberoaméricano de Informatica Educativa. Colombia 1996.

- B. Kozma. Learning with media. Review of Educational Research, Summer 1991, vol. 61. $N^{\circ} 2$, pp 179-211. 